\title{
Evaluating User Experience of Augmented Reality-Based Automobile Maintenance Content - Mobile Device and HoloLens Comparison -
}

\author{
Myeong-Hyeon Heo, Dongho Kim and Jaewoon Lee \\ Department of Information Communication, Materials, and Chemistry \\ Convergence Technology, Soongsil University \\ Department off Digital Media, Soongsil University \\ jwlee@gsclab.kr
}

\begin{abstract}
Augmented visualization was an earlier form of augmented reality and it utilized desktop computers and mobile devices. It has evolved to the glass form of augmented reality such as Google Glass and HoloLens. It is predicted that the new development will provide more intuitive interaction and both hands will be used actively in the new work environment. The automobile industry has combined the advanced technology and software programs to provide various functions. Moreover, it has introduced new types of automobiles to the market including eco-friendly automobiles. These developments have made the structure and function of automobiles more complex and the design and option of them more diverse. However, there are not enough skilled technicians to cope with these changes. This problem can be resolved if the technical document, database, and $3 D$ sensing information are overlaid on the actual objects precisely using the HoloLense technology, which is based on augmented reality technology. Delivering the necessary information in real time will make the automobile maintenance easier and it will assist automobile technicians to maintain automobiles more intuitively and safely. Obtaining the necessary skills will also be easier with less effort and training. Moreover, it will eventually increase customer satisfaction because it will help manufacturers and automobile maintenance service companies allocate service personnel more efficiently and handle various situations with fewer people. The objective of this study was to provide necessary baseline data for developing future services by evaluating and analyzing the user experiences of augmented reality contents using mobile devices and HoloLens for maintaining automobile better in the future. This study realized three augmented as a preliminary study for evaluating the user experience necessary for developing augmented reality contents for automobile maintenance. This study conducted the user experience evaluation after having subjects experience these three contents using the mobile device or HoloLens.
\end{abstract}

Keywords: Augmented Reality, HoloLens, Automobile Maintenance, User Experience

\section{Introduction}

Augmented visualization was an earlier form of augmented reality and it utilized desktop computers and mobile devices. It has evolved to the glass form of augmented reality such as Google Glass and HoloLens. It is predicted that the new development will provide more intuitive interaction and both hands will be used actively in the new work environment [1] [2].

The automobile industry has combined the advanced technology and software programs to offer various functions. Moreover, it has introduced new types of

Received (December 28, 2017), Review Result (February 20, 2018), Accepted (February 24, 2018) 
automobiles to the market including eco-friendly automobiles. These developments have made the structure and function of automobiles more complex and the design and option of them more diverse. However, the demand for skilled technicians has become beyond the supply [3]. This problem can be resolved if the technical document, database, and 3D sensing information are overlaid on the actual objects precisely using the HoloLense technology, which is based on augmented reality technology. Delivering the necessary information in real time will make the automobile maintenance easier. It will assist automobile technicians to maintain automobiles more naturally and safely. Obtaining the necessary skills will also be easier with less effort and training. Moreover, it will eventually increase customer satisfaction because it will help manufacturers and automobile maintenance service companies allocate service personnel more efficiently and handle various situations with fewer people.

Numerous studies evaluated the interaction between trainees and implemented augmented reality-based mobile devices. The results of these studies showed that the augmented reality technology could stimulate the potential and interest of learners [4][5]. However, only a few analyses have been conducted regarding the user experience of HoloLens, which is extraordinarily improved augmented reality equipment. The objective of this study was to help the development of an effective service in the future by comparing and analyzing the user experience of augmented reality contents between mobile devices and HoloLens in maintaining automobiles.

\section{Related Studies}

Unlike virtual reality, augmented reality superimposes a virtual object on the real space. It has an advantage of providing supplementary information, which is hard to acquire in the real world, by mixing two types of information [6]. Azuma (1997) stated that there are three requirements for implementing the augmented reality. First, it shall create a seamless world by combining the real world and the virtual world. Secondly, it shall allow a user and a system to communicate continually in real time. Thirdly, a virtual object shall be superimposed on the 3D space precisely to please the five senses of a user and enhance immersion feeling. Azuma (1997) claimed that augmented reality is not achieved just by merging a real world and a virtual world. Azuma (1997) also argued that it could be considered augmented reality only when two merged elements mutually circulate and have an organic relationship [7].

Billinghurst (2002) argued that augmented reality has five advantages. First, it combines the real-world element with a virtual image to give a smooth interaction in real time. Secondly, it presents information more than one can see and enhances the sense of reality by combining the elements of reality with virtual information, another reality. Thirdly, it provides a user with enhanced digital information and augmented reality in the virtual environment merged with a real environment, beyond the spatial constraints. Fourthly, it helps a user give a new metaphor to an interface. For example, a user can use an object in the environment as a digital medium, beyond the common operation, using the mouse and keyboard, the traditional input devices of the computer. Fifthly, it creates a smooth transition from the real world to the virtual world [8].

The types of the augmented reality display can be divided into "See-through Head Mounted Device", "Projection-based Display", and "Handheld-based Display". The "Seethrough Head Mounted Device" is the most similar to the way that the human beings watch objects. However, it has to be worn on the head and the angle of view is limited. The "Projection-based Display" has an advantage that multiple people can watch at the same time. However, it has a limited mobility. Furthermore, the field of view and the distance of view are restricted according to the disposition of seats. The "Handheld-based Display" type has been currently developed and advanced the most owing to the increase of smartphones and it has high mobility. Depending on the location, augmented reality 
can be classified into stationary augmented reality and mobile augmented reality. "Seethrough Head Mounted Device" and "Handheld-based Display", among the display classification, are the mobile type and "Projection-based Display" represents the stationary type.

As the technology based on head mounted display (HMD) have advanced, Microsoft recently released HoloLens, which can be used without using mobile devices or desktop computers. HoloLens has given augmented reality new possibilities because it matches a created 3D model with a real space by recognizing the characteristics of an environment and can save it in the actual space. Especially, it is possible to produce immersive and highly interactive contents because the HMD-based augmented reality is not restricted by screen and space [9][10].

\section{Experimental Methods}

\subsection{Subjects of Experiment}

This study conducted an experiment on 44 undergraduate students who were majoring in Automotive and Mechanical Engineering in a college located in Seoul and not exposed to HoloLens. The subjects were divided into two groups (22 subjects each). The study treated the mobile device usage and the HoloLens usage as independent variables and the evaluation score after the usage as a dependent variable.

\subsection{Experiment Procedure}

\section{Table 1. Resolution of Mobile Devices \& HoloLens and Performance of Camera}

$\begin{array}{lll}\text { Model Samsung Galaxy Note8 } & \text { MS HoloLens }\end{array}$

\begin{tabular}{llll}
\hline Image & $2960 \times 1440($ Quad HD+) \\
\hline Resolution & 12 million pixels & \\
\hline Camera & & \\
\hline
\end{tabular}


Table 2. Augmented Reality Contents

\begin{tabular}{lll}
\hline Classification & Image & Effect \\
\hline Content A & 3D Model & Silhouette \\
\hline Content B & Label \\
\hline Content C & & \\
\hline
\end{tabular}

This study created virtual objects of three contents by using Unity 3D [11] and Vuforia SDK [12] based on literature review and existing augmented reality cases.

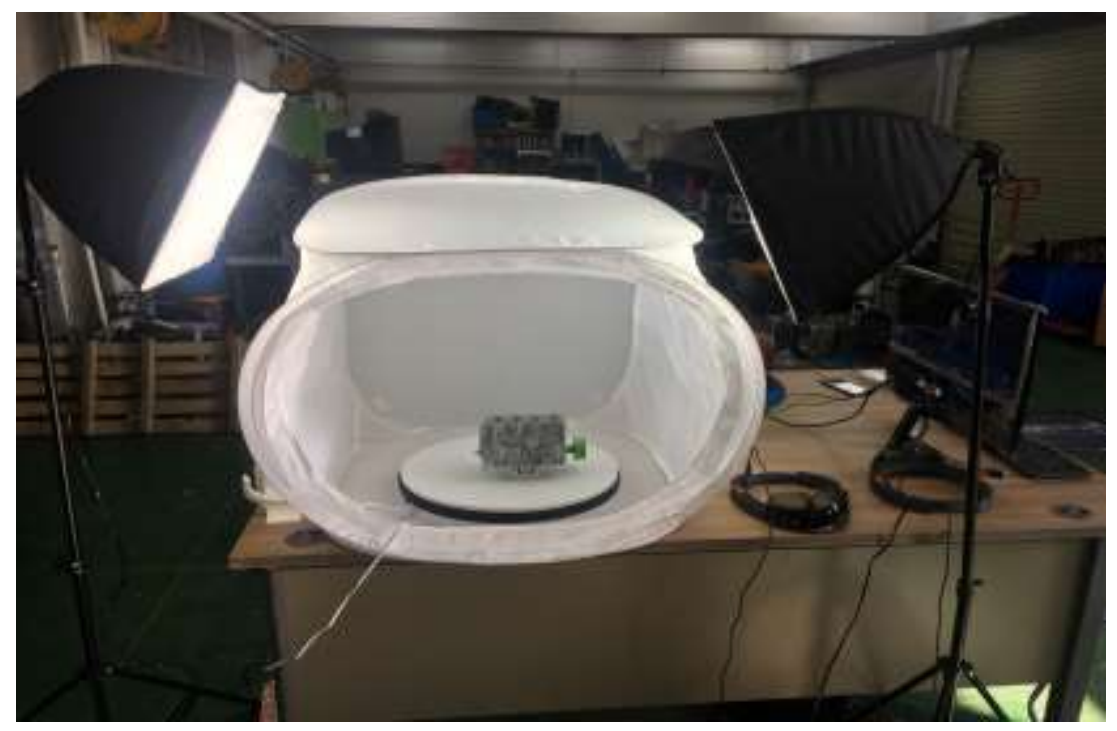

Figure 1. Experimental Environment 
Contents $\mathrm{A}$ and $\mathrm{B}$ expressed the piston structure and motions in an engine miniature model by animation. Content $\mathrm{A}$ used an opaque 3D model and content $\mathrm{B}$ applied a silhouette effect so that the engine miniature model is not hidden by the content. Content $\mathrm{C}$ displayed the name of a part using a label after recognizing the accurate position of the piston without using an animation effect [Table. 2].

The developed augmented reality contents (i.e., virtual objects) were observed in a studio furnished with two stand lamps with considering the limitations of the hardware of HoloLens developed so far in order to improve the experiment condition [Figure 1].

For evaluating the user experience, the user group using a mobile device grasped the device with one hand and touched the screen to operate the object [Figure 1](a). The user group using HoloLens operated the device with gesture and voice command to evaluate the user experience [Figure 1](b).

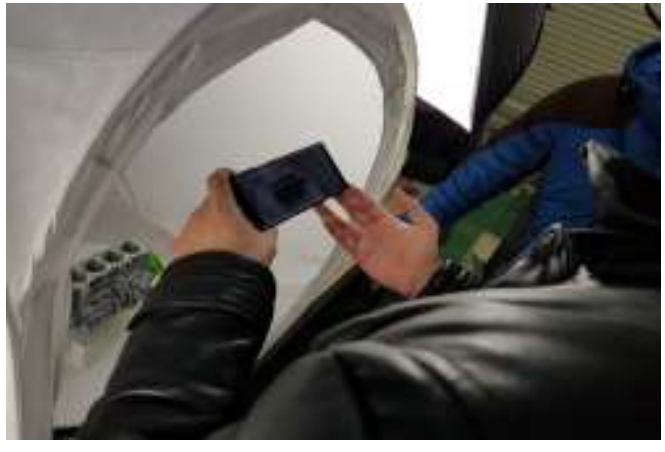

(a)

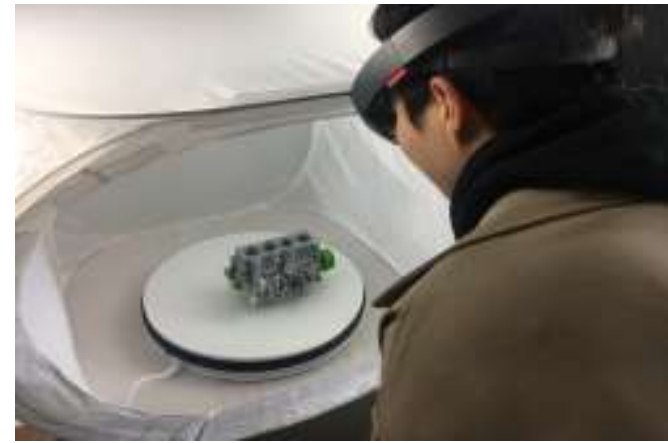

(b)

Figure 2. (a)Test for Mobile Device, (b) Test for HoloLens

The user evaluation was made with referring to the questionnaire of Park and Kim (2017)[13]. The questionnaire consisted of four items (i.e., awareness, comfort, functionality, and space perception). Respondents were asked to answer in five-point Likert scale (i.e., very poor, poor, average, good, and very good).

Table 3. Evaluating Items for Usability and the Explanations

\begin{tabular}{|c|c|}
\hline Classification & Description \\
\hline Awareness & $\begin{array}{l}\text { GUI element and degree of recognizing } \\
\text { information }\end{array}$ \\
\hline Comfort & Comfort of eyes in the visual aspect \\
\hline Functionality & Structural stability or clarity of distinction \\
\hline Space Perception & Distinction (depth) between real and virtual objects \\
\hline
\end{tabular}

\subsection{Data Processing}

Statistical analyses were conducted using IBM SPSS Statistics 24. In order to test the normality of the mobile device user group and the HoloLens user group, KolmogorovSmirnov was conducted. The results of Kolmogorov-Smirnov indicated that these groups did not satisfy the assumptions of normality $(p>0.05)$. Therefore, this study conducted Mann-Whitney test, a non-parametric test, to compare these two groups. 


\section{Results}

Table 4. Evaluation Results of User Experiences (A)

\begin{tabular}{|c|c|c|c|c|}
\hline Classification & Group (N) & $\begin{array}{c}\text { Awareness } \\
(\text { Mean } \pm \text { SD) }\end{array}$ & $\mathbf{Z}$ & p-value \\
\hline \multirow{2}{*}{ Awareness } & Mobile Device (22) & $4.40 \pm .66$ & \multirow{2}{*}{-1.655} & \multirow{2}{*}{.098} \\
\hline & HoloLens (22) & $3.86 \pm 1.08$ & & \\
\hline \multirow{2}{*}{ Comfort } & Mobile Device (22) & $3.59 \pm 79$ & \multirow{2}{*}{-.854} & \multirow{2}{*}{.393} \\
\hline & HoloLens (22) & $3.40 \pm 73$ & & \\
\hline \multirow{2}{*}{ Functionality } & Mobile Device (22) & $3.86 \pm .99$ & \multirow{2}{*}{-.437} & \multirow{2}{*}{.662} \\
\hline & HoloLens (22) & $3.72 \pm 1.03$ & & \\
\hline \multirow{2}{*}{$\begin{array}{c}\text { Space } \\
\text { Perception }\end{array}$} & Mobile Device (22) & $3.68 \pm 71$ & \multirow{2}{*}{-.447} & \multirow{2}{*}{.655} \\
\hline & HoloLens (22) & $3.86 \pm 83$ & & \\
\hline
\end{tabular}

Table 6 Evaluation Results of User Experiences (B)

\begin{tabular}{|c|c|c|c|c|}
\hline Classification & $\operatorname{Group}(\mathbf{N})$ & $\begin{array}{l}\text { Awareness } \\
(\text { Mean } \pm \text { SD) }\end{array}$ & $\mathbf{Z}$ & p-value \\
\hline \multirow{2}{*}{ Awareness } & Mobile Device (22) & $3.36 \pm 1.04$ & \multirow{2}{*}{-.086} & \multirow{2}{*}{.932} \\
\hline & HoloLens (22) & $3.36 \pm 1.00$ & & \\
\hline \multirow{2}{*}{ Comfort } & Mobile Device (22) & $3.68 \pm .94$ & \multirow{2}{*}{-2.380} & \multirow{2}{*}{.017} \\
\hline & HoloLens (22) & $3.00 \pm .87$ & & \\
\hline \multirow{2}{*}{ Functionality } & Mobile Device (22) & $3.09 \pm 1.01$ & \multirow{2}{*}{-.512} & \multirow{2}{*}{.608} \\
\hline & HoloLens (22) & $3.32 \pm .78$ & & \\
\hline \multirow{2}{*}{$\begin{array}{c}\text { Space } \\
\text { Perception }\end{array}$} & Mobile Device (22) & $3.23 \pm .97$ & \multirow{2}{*}{-.414} & \multirow{2}{*}{.679} \\
\hline & HoloLens (22) & $3.18 \pm .95$ & & \\
\hline
\end{tabular}

Table 7. Evaluation Results of User Experiences(C)

\begin{tabular}{|c|c|c|c|c|}
\hline Classification & Group (N) & $\begin{array}{l}\text { Awareness } \\
(\text { Mean } \pm \text { SD) }\end{array}$ & $\mathbf{Z}$ & p-value \\
\hline \multirow{2}{*}{ Awareness } & Mobile Device (22) & $3.95 \pm .72$ & \multirow{2}{*}{-.025} & \multirow{2}{*}{.980} \\
\hline & HoloLens (22) & $3.86 \pm 1.08$ & & \\
\hline \multirow{2}{*}{ Comfort } & Mobile Device (22) & $3.82 \pm .73$ & \multirow{2}{*}{-.621} & \multirow{2}{*}{.535} \\
\hline & HoloLens (22) & $3.64 \pm .90$ & & \\
\hline \multirow{2}{*}{ Functionality } & Mobile Device (22) & $3.59 \pm .95$ & \multirow{2}{*}{-.200} & \multirow{2}{*}{.842} \\
\hline & HoloLens (22) & $3.64 \pm 1.00$ & & \\
\hline \multirow{2}{*}{$\begin{array}{c}\text { Space } \\
\text { Perception }\end{array}$} & Mobile Device (22) & $3.45 \pm .96$ & \multirow{2}{*}{-.941} & \multirow{2}{*}{.941} \\
\hline & HoloLens (22) & $3.48 \pm .95$ & & \\
\hline
\end{tabular}


The evaluation results of user experiences for content A revealed that the awareness of the mobile device group was $4.40 \pm .66$ and that of the HoloLens group was $3.86 \pm 1.08$. Although the former was higher than the latter, they were not significantly different

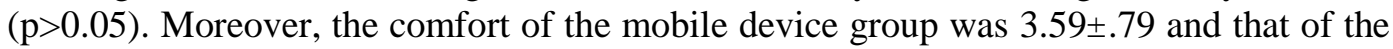
HoloLens group was 3.40土.73. Even though the former was higher than the latter, there was no significant difference between them $(p>0.05)$. The functionality of the mobile

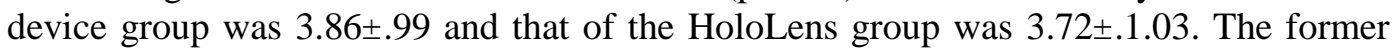
was higher than the latter but they were not significantly different $(p>0.05)$. The space perception of the mobile device group was 3.68 71 and that of the HoloLens group was $3.86 \pm .83$. Although they were not significantly different $(p>0.05)$, the former was higher than the latter.

The evaluation results of user experience for content B showed that the awareness of the mobile device group was 3.36 \pm 1.04 and that of the HoloLens group was 3.36 \pm 1.00 . The former was higher than the latter but there was no significant difference between them ( $p>0.05$ ). Moreover, the comfort of the mobile device group was 3.68 .94 and that

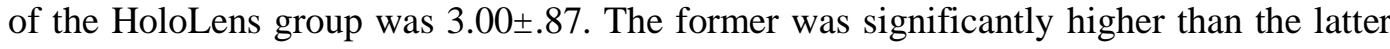
$(\mathrm{p}<0.05)$. The functionality of the mobile device group was $3.09 \pm 1.01$ and that of the HoloLens group was 3.32 \pm .78. Although the former was higher than the latter, they were not significantly different ( $p>0.05$ ). The space perception of the mobile device group was

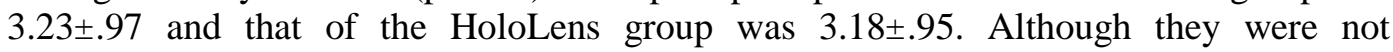
significantly different $(\mathrm{p}>0.05)$, the former was higher than the latter.

The evaluation results of user experiences for content $\mathrm{C}$ indicated that the awareness of

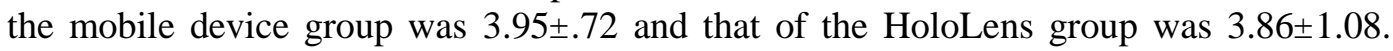
Although the mobile device group showed higher awareness than the HoloLens group, they were not significantly different ( $p>0.05$ ). Additionally, the comfort of the mobile

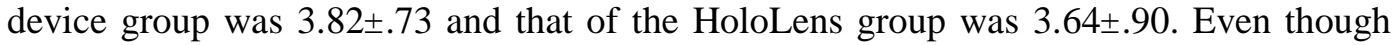
the former was higher than the latter, there was no significant difference between them ( $>0.05$ ). The functionality of the mobile device group was $3.59 \pm .95$ and that of the HoloLens group was 3.64 1.00 . The latter was higher than the former but they were not significantly different $(\mathrm{p}>0.05)$. The space perception of the mobile device group was $3.45 \pm .96$ and that of the HoloLens group was 3.48土.95. Although the latter was higher than the former, they were not significantly different $(\mathrm{p}>0.05)$.

\section{Conclusion and Future Work}

This study materialized three contents as a preliminary study for evaluating the user experience necessary for developing augmented reality contents for automobile maintenance. Study subjects experienced the contents using the mobile device or HoloLens and conducted the user experience evaluation. The results of this study showed that the user experiences were not significantly different between the mobile device and the HoloLens except for the comfort of content B. The awareness of content A showed the largest difference between the mobile device group and the HoloLens group. It could be due to the limitation of the HoloLens hardware. On the other hand, the space perception of HoloLens was better than that of the mobile device for content A and B despite the difference in resolution and camera performance of two devices. It was believed that it was because HoloLens offered a superior immersion performance as an HMD device. It was speculated that there are no clear differences between comfort and functionality between two tested devices because the augmented reality contents used in this study presented the structure of the piston in an automobile engine miniature and users focused on the structural information of the piston too much.

When the results of user experience evaluation were compared, the content A had the highest scores in awareness, functionality, and space perception while the content $\mathrm{B}$ received the highest score in comfort. The results of this study implied that the 3D model 
animation of the content $\mathrm{A}$ helped subjects recognize information better, increased the clarity of information, and enhanced the difference between the reality and the virtual objects. On the other hand, subjects considered the content $\mathrm{C}$ as the most visual-friendly because it displayed the name and position of a part on a fixed image. The results of this study suggested that the usability of contents might be maximized when the advantages of three contents are mixed together rather than determining that the content $\mathrm{A}$ is the best.

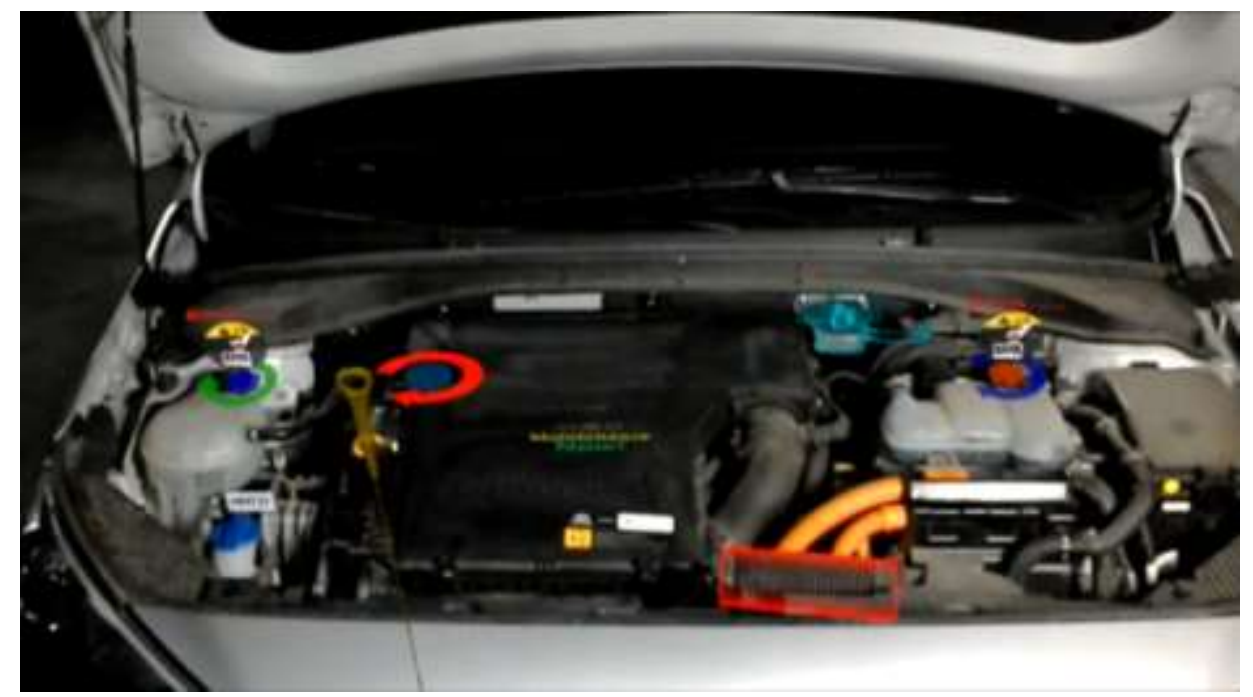

\section{Figure 3. Application of Augmented Reality Content to the Hybrid Automobile Engine Room}

We will continuously improve and supplement the augmented reality-based automobile maintenance contents based on the feedback of the learners by testing the contents in the field repeatedly. It will be required to develop visualization method considering various automobile maintenance situations in order to conquer the limitations of the existing HoloLens hardware. Particularly, this study confirmed that the HoloLens had a restraint in tracking objects for each frame. It will be necessary to study ways of matching 3D models in the real environment to overcome the limitation. It was predicted that the HoloLens type device is in its early stage, compared to the mobile device, and it will provide better user experiences if the performance of it is enhanced in the future.

\section{Acknowledgments}

This paper is a revised and expanded version of a paper entitled Asia-pacific Journal of Convergent Research Interchange presented at December 17, 2017, Namseoul University, Cheonan, Korea.

This work was supported by Institute for Information \& communications Technology Promotion(IITP) grant funded by the Korea government(MSIP) (No. 2017-0-01785, The Development of Automotive Maintenance Framework).

\section{References}

[1] S. Ho Choi, M. Hun Lee and J. Yeol Lee, "A Comparative Analysis of User Experience in Home Energy Saving Awareness Using Immersive Virtual Reality and Mobile Augmented Reality”, Korean Journal of Computational Design and Engineering, vol. 21, no. 4, (2016), pp. 397-408.

[2] M.-H. Heo, E.-Y. Lee and D. Kim, "A Verification of the Effectiveness of Spatial Augmented Realitybased CCA for the Improvement of Traditional Sports Climbing Lessons", Journal of The Korea Contents Association, vol. 17, no. 5, (2017), pp. 90-99.

[3] S. Park, V. Tran Hoang, A. Nguyen Hoang, G. Bae, J. Lee and D. Kim, "Automobile Maintenance Training System using Phased Learning based on Virtual Reality", Journal of KIISE : Computing Practices and Letters, vol. 19, no. 12, (2013), pp. 663-667. 
[4] H.-K. Wu, "Current status, opportunities and challenges of augmented reality in education", Computers \& Education, vol. 62, (2013), pp. 41-49.

[5] J. Martín-Gutiérrez, "Augmented reality to promote collaborative and autonomous learning in higher education", Computers in Human Behavior, vol. 51, (2015), pp. 752-761.

[6] P. Milgram and F. Kishino, "A taxonomy of mixed reality visual displays", IEICE Transactions on Information and Systems, vol. 77, no. 12, (1994), pp. 1321-1329.

[7] R. T. Azuma, “A survey of augmented reality”, Presence: Teleoperators and virtual environments, vol. 6, no. 4, (1997), pp. 355-385.

[8] M. Billinghurst, "Augmented reality in education", New horizons for learning, vol. 12, (2002).

[9] S. Kim, S. Young Han and J. Kim, "Hololens-based Immersive Interactive Storytelling Design: From "Sit \& Watch" to "Walk \& Experience"”, HCI Korea 2018, (2017), pp. 425-428.

[10] H.-y. Kim, "The Current Status and Development Direction of Mixed Reality Content", Catoon \& Animation Studies, vol. 46, (2017) March, pp. 181-206.

[11] https://unity3d.com/kr, (2017)November 1 .

[12] https://www.vuforia.com, (2017) November 1.

[13] J. Hong Park and H. Kim, "Research on GUI(Graphic User Interface) Design Styles in Mixed Reality Service - Focusing on the Way to Distinguish between Reality and Virtual Object", Journal of The Korean Society Design Culture, vol. 23, no. 2, (2017), pp. 271-282.

\section{Authors}

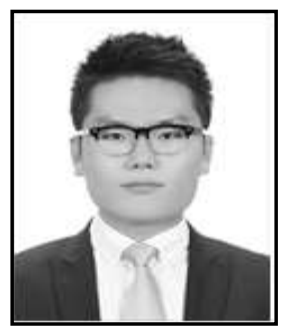

Myeong-Hyeon Heo is a Ph.D. candidate student at GSC Lab., in the Department of Information Communication, Materials, and Chemistry Convergence Technology at Soongsil University, Seoul, Korea. He received his Master degree in Sports Information Technology at Soongsil University in 2014. His interests include motor learning and mixed reality. Contact him at heomaeng@gsclab.kr

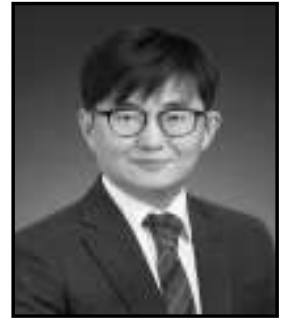

Dongho Kim is a Professor in the Department of Digital Media at Soongsil University, Seoul, Korea. He obtained B.Sc. degree from Seoul National University and Master degree from KAIST, Korea. $\mathrm{He}$ received Ph.D. degree in Computer Science at the George Washington University in 2002. His research interests include realtime rendering, animation, game engineering, digital contents, and media art. Contact him atcg@ssu.ac.kr

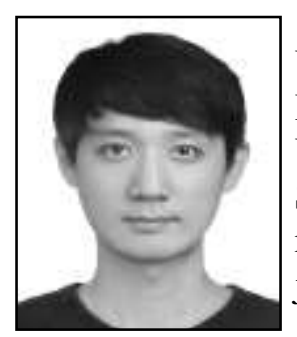

Jaewoon Lee is a Research Fellow at GSC Lab in Soongsil University, Seoul, Korea. He obtained B.A. degree from Seoul Instituted of the Arts and Master degree from Soongsil University, Korea. He received Ph.D. in Media Art from Soongsil University in 2017. His research interests include mixed reality, interactive contents, and media art. Contact him at jwlee@gsclab.kr. 
International Journal of Control and Automation

Vol. 11, No. 4 (2018) 